\title{
Analysis for Influence of Excavation on Adjacent Existing Shield Tunnel
}

\author{
Jing Cao* \& Guowei Qian \\ School of Architectural and Civil Engineering, Kunming University of Science and Technology, Kunming \\ 650500, China
}

Haixi Zuo

CABR Foundation Yunnan Co., Ltd., Kunming 650016, China

Haiming Liu

School of Architectural and Civil Engineering, Kunming University of Science and Technology, Kunming 650500, China

Yaliang Ren

Beijing Urban Construction Design and Development Group Co., Ltd, Xi'an 710016, China

ABSTRACT: The horizontal earth pressure of shield tunnel affected by excavation was analyzed. Firstly, the foundation pit excavation disturbance zone boundary and the strain of level unit soil in disturbance zone were assumed. Meanwhile, the formula for calculating the horizontal earth pressure in the disturbed zone of the pit is derived. Afterwards, combined with the proposed earth pressure theory and soil arch theory, the model of horizontal earth pressure of shield tunnel consider the affected by foundation pit excavation was established. Finally, joining an engineering example, verify the rationality of the basic assumptions and theoretical deduction. The result shows that when the tunnel is located in the disturbed zone of the foundation pit, surrounding rock pressure on both sides of the tunnel will be effected, and the sides of shield tunnel near foundation pit will be larger influenced by excavation.

KEYWORD: Excavation; Existing shield tunnel; Soil arch effect; Pit outer disturbed zone; Surrounding rock pressure

\section{INTRODUCTION}

With the rapid development of economy in our country, rail transit construction boom is sweeping across the country large and medium-sized cities. Because of subway effect phenomenon, the land price is soaring in along metro lines. Usually, almost all the land development after rail transit construction had finished, so it can't be avoided to encounter some difficulties that foundation pit excavation around the shield tunnel. Obviously, foundation pit excavation will affect the adjacent existing shield tunnel security, such as aggravated deformation of tunnel lining structure, generated the whole settlement or lateral, etc. However, deformation and displacement of shield tunnel need fairly strict control in Subway Engineering. Hence, it is an urgent problem that analysis on mechanism of Existing Metro Shield Tunnel impacted by excavation of deep foundation pit.

Currently, many domestic and international scholars have devoted to the research work, and also obtained large number of achievements in theoretical research and engineering application. Generally, it research methods including measured data analysis (Ding Yongchun et al, 2008) (Wei Gang et al, 2013), centrifugal model test analysis (Ling Hao et al, 2010) (Liang Fayun et al, 2012), two-stage method (Zhang Zhiguo et al, 2011) (Huang Xiang et al, 2012) and
Finite element method (Wang Yanyan et al, 2011) (Shen Hui et al, 2011) (Xu Huang et al, 2013).In a word, mainly through fitting and the finite element method to study of law between tunnel deformation and foundation pit excavation, tunnel deformation control measures and foundation pit supporting scheme evaluation at the present stage. However, though earth pressure calculation of shield tunnel is a core problem to analyze it deformation mechanism, still few scholars study it. For this reason, based on assumptions of disturbed area by excavation, horizontal strain in the zone and the soil arch theory, presented in this article that the calculation method of existing tunnel horizontal soil pressure, and it has clear concept and convenient operation.

\section{FUNDAMENTAL ASSUMPTIONS}

\subsection{The active disturbance area boundary assumption.}

In the classical theory of slip, within critical slip of outside foundation pit soil affected by excavation and outside the area stable. Nevertheless, it is possible to the outside critical slip soil impacted by excavation for practical engineering. 


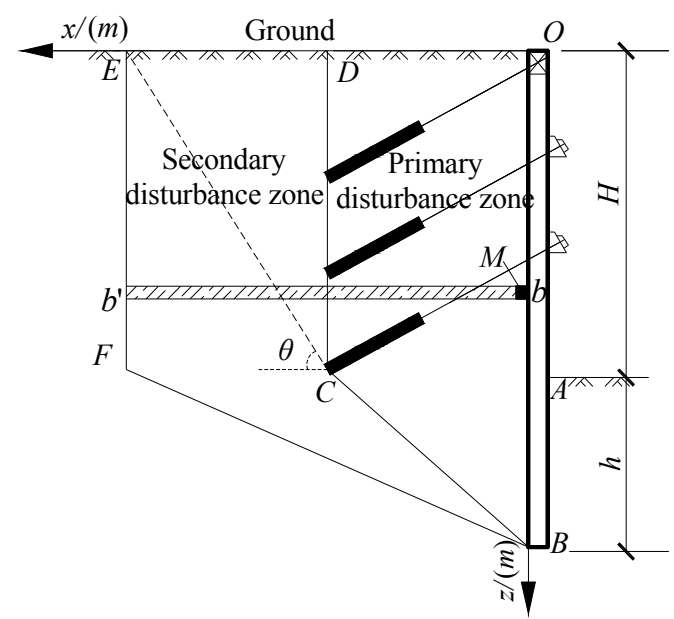

Fig. 1 The disturbance zone sketch in pile anchorage foundation pit

So based on the KRANZ Method (Zhang Limin et al, 1992) (Zhou Mengjia et al, 2011) (Wang Ning, 2008), and made assumptions that surface of BCD in Fig. 1 for main disturbance zone boundary to pile anchor foundation pit, it constructs the main disturbance zone together with retaining pile. Simultaneously, point B is the bottom of retaining piles, point $\mathrm{C}$ is located in the last row anchor cable endpoint, and point $\mathrm{D}$ is the projection of the $\mathrm{C}$ point on the natural ground. Next, under the action of anchor bolt, this soil within the BCD plane will be produced slip deformation together with the foundation pit system, so this soil of after CD will be disturbed. According to classical earth pressure theory, it will be formed a slip planes that the angle with horizontal direction is $45^{0}+\varphi / 2$. Thus, the active slip plane in pile anchor foundation pit could be simplified as BCF. But, also considering the existence influence factors such as time effect, space effect and surrounding environment in excavation, led to outside BCE areas still could be disturbed by excavation and also should not be ignored it. Therefore, combined with related theories assumed that the excavation disturbed zone boundary surface for BFE on the premise of easy to deduce, it shown in Fig. 1, and point $\mathrm{F}$ and point $\mathrm{E}$ in the same vertical line as well as point $\mathrm{F}$ and $\mathrm{C}$ at the same level. At the same time, this region surrounded by BFE and supporting regional is called disturbance zone.

\subsection{Assumption of horizontal strain distribution.}

As show in Fig. 1, a soil element $b b^{\prime}$ that arbitrary selection in excavation disturbed zone is analyzed. At this point, point $b$ is contact with foundation pit supporting structure, and point $b^{\prime}$ is located at BFE surface. Meanwhile, it is assumed that the deformation compatibility is satisfied between supporting structure and soil body. That is, between supporting structure and soil is never separated in the excavation, and supporting structure deformation $v(z)$ is equal to unit earth $b b^{\prime}$ deformation in point $b$, which is each point deformation accumulation. As show in Fig. 2, the article make a assumption that the strain along the horizontal direction is triangular $(\mathrm{Ou}$ Mingxi et al, 2012), any point horizontal strain expressions is shown in the formula (1).

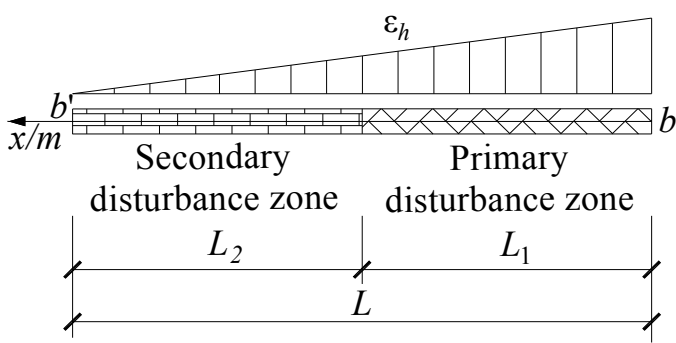

Fig. 2 The unit soil bb' horizontal strain distribution sketch

$\varepsilon_{h}=\frac{2(L-x)}{L^{2}} v(z)$

Where, $\varepsilon h$ is the horizontal strain of any point in disturbance zone. $L$ is the horizontal distance that disturbance zone boundary to the supporting structure in any depth $z, \mathrm{~m} . x$ is the horizontal distance calculating point to supporting structure, m. $v(z)$ is the horizontal deformation of retaining structure in any depth $z, \mathrm{~m}$.

\subsection{Tunnel disturbance criterion.}

Based on the above assumptions, the criterion for judge the excavation wether impact on the adjacent shield tunnel was proposed. When the existing shield tunnel didn't enter the disturbanced zone by excavation, can consider that the foundation pit excavation haven't any effect on it, and the calculation of surrounding rock pressure still take into account soil arch effect. On the contrary, when the existing shield tunnel enter the disturbanced zone by excavation, the original stress balance will be destroyed by excavation, thus tunnel rock pressure will be changed.

\section{MECHANICAL MODEL AND DERIVATION}

\subsection{Foundation pit earth pressure calculation model.}

As show in Fig.1, at point $b$, interception unit soil $\mathrm{M}$ for the research object. Research shows that during excavation process vertical earth pressure $\sigma_{a k}$ of unit soil $\mathrm{M}$ is remain unchanged, but horizontal earth pressure $p_{a k}$ is reduced gradually due to impressed by excavation. Generally, when supporting structure undeformed, the horizontal earth pressure of unit soil $\mathrm{M}$ is static earth pressure will remains unchanged, and denoted as $p_{a o}$. When deformation of supporting structure toward foundation pit, horizon- 
tal pressure of the unit $\mathrm{M}$ is reduced gradually with it increasing, and the reduction is called $\Delta p_{a k}$. Thus, horizontal earth pressure of unit soil $\mathrm{M}$ calculation formula is as follow.

$$
p_{a k}=p_{a o}-\Delta p_{a k}
$$

Where, $p_{a k}$ is horizontal earth pressure of unit soil $\mathrm{M}, \mathrm{kN} / \mathrm{m}^{2}$, and when it is less than zero, taken it as zero. $p_{a 0}=\gamma z K_{0}$ is called static earth pressure that acted at any depth of supporting structure, $\mathrm{kN} / \mathrm{m}^{2} . \gamma$ is called unit weight of soil, $\mathrm{kN} / \mathrm{m}^{3} . z$ is depth of calculated point to natural ground, m. $K_{0}=1-\sin \varphi^{\prime}$ is static earth pressure coefficient. $\varphi^{\prime}$ is the effective angle of internal friction of soil, ${ }^{\circ} . \Delta p_{a k}$ is the reduction horizontal pressure due to supporting structural deformation $v(z)$ impressed by excavation, $\mathrm{kN} / \mathrm{m}^{2}$. According to Winkler assumption, establish $\Delta p_{a k}$ relationship with $v(z)$ for $\Delta p_{a k}=k_{a k} \cdot v(z)$. Then, take it substitute it into formula (2), the formula for calculating the horizontal earth pressure of unit soil $\mathrm{M}$ will be achieved as follow.

$$
p_{a k}=p_{a o}-k_{a k} \cdot v(z)
$$

Where, $k_{a k}$ is equivalent horizontal earth pressure resistance coefficient, $\mathrm{kN} / \mathrm{m}^{3} . v(z)$ is horizontal deformation of support structure for depth $z, \mathrm{~m}$.

Based on the Rankine's earth pressure theory, when $v(z)=v_{a}$, the soil pressure acting on retaining structure is active earth pressure, namely $p_{a k}=\sigma_{a}=\gamma z K_{a}-2 c \sqrt{K_{a}}$, which $K_{\mathrm{a}}=\tan ^{2}\left(45^{\circ}\right.$ $\varphi / 2), v_{a}$ is deformation of supporting structure when active limit equilibrium state is reached in Rankine active destroying. Literature (Zai Jinmian et al, 2002) present when the soil is cohesionless soils, $v_{a}=(0.003 \sim 0.005) H$; But, when it is cohesive soil, $v_{a}=(0.004 \sim 0.010) H$. Where, $H$ is foundation pit excavation depth, m. Next, substitute the boundary conditions into formula (3), can solve the undetermined coefficient $k_{a k}$ in formula (3).

$k_{a k}=\frac{\gamma z\left(K_{0}-K_{a}\right)+2 c \sqrt{K_{a}}}{v_{a}}$

\subsection{The soil pressure calculation in disturbance zone.}

As shown in Figure 2, the deformation of point $b^{\prime}$ is 0 , and point $b$ is $v(z)$. By deformation and strain relationship, can get the calculation formula (5) of horizontal deformation at any point $x$ in unit soil slices.

$v(z, x)=\int_{x}^{L} \varepsilon_{h} d x=\left(1-\frac{2 L x-x^{2}}{L^{2}}\right) v(z)$
Let to $\delta_{h}=1-\frac{2 L x-x^{2}}{L^{2}}$, and $\delta_{h}$ is horizontal displacement influence coefficient. So formula (5) can be rewritten as $v(z, x)=\delta_{h} \cdot v(z)$, where $v(x, z)$ is horizontal deformation to any point $(x, z)$ in unit soil slices, m. Let's plug equation (4) and (5) into equation (3), obtained in the derivation process is calculation formula of earth pressure at any point in disturbed zone.

$$
p_{a k}^{\prime}=p_{a 0}-k_{a k} \cdot \delta_{h} \cdot v(z)
$$

Where, $p^{\prime}{ }_{a k}$ is horizontal earth pressure at any point $x$ in unit soil slices, $\mathrm{kPa}$, in the same way, when it less than 0 , take it as 0 . Thus, other symbols in formula (6) are similar with the former.

\subsection{The calculation of surrounding rock pressure before excavation.}

Before excavation, the shield tunnel is in a state of static equilibrium, and the calculation of surrounding rock pressure can be implemented by the method described in reference (Zhu Hehua, 2010), and the concrete analyses arefollowing.

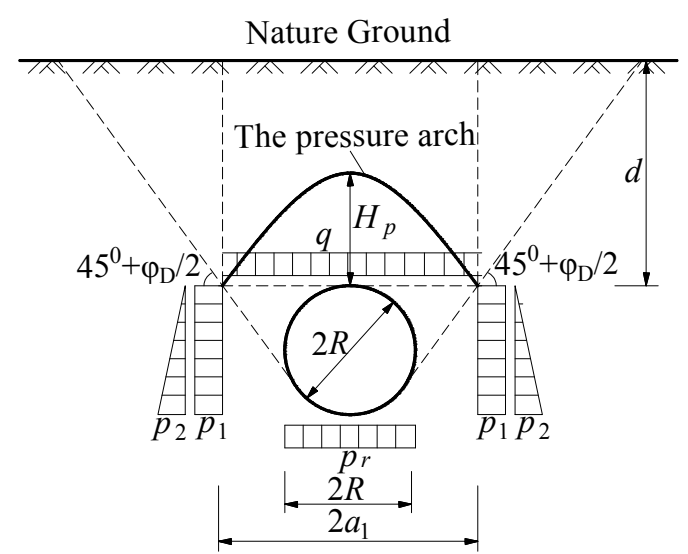

Fig. 3 The shield tunnel calculation diagram before the excavation

Firstly, the vertical rock pressure at the top of shield tunnel is analyzed. As described in the reference (Zhu Hehua, 2010), when shield tunnel was buried in soft clay layer, the vertical surrounding rock should be according to all the top soil pressure calculation. At the same time, when shield tunnel is buried in the soil has a large shear strength and buried depth over the shield outside diameter $(d>2 R)$, need to consider the soil arch effect, so the vertical surrounding rock less than all the top soil pressure, it can be calculated according to the theory of loose height. Besides, application of Protojiakonov's to calculate the loose height, as follows formula (7).

$$
h_{p}=\frac{a_{1}}{f_{k}}
$$


Where, $h_{p}$ is high pressure arch of tunnel roof, $\mathrm{m}$. $f_{k}$ is Protodyakonov coefficient, $f_{k}=\tan \varphi_{D}$, and $\varphi_{D}$ is angle of equivalent internal friction, according to the method in reference (Cao Jing et al, 2015) to calculated, that is, $\varphi_{D}=\arctan \left(\tan \varphi+\frac{c}{\sigma}\right), \varphi$ is angle of internal friction, ${ }^{\circ} . \sigma$ is normal stress on the shear plane, $\mathrm{kPa}$, other symbols with the former.

In a word, when not consider soil arch effect in the roof of tunnel, the calculation formula of shield tunnel vertical rock pressure as follows.

$q=\gamma_{m} d+q_{0}$

Where, $q$ is vertical rock pressure in the roof of shield tunnel, $\mathrm{kPa} . \gamma_{m}$ is the weighted average that weight per unit volume of soil layer thickness above the tunnel roof, $\mathrm{kN} / \mathrm{m}^{3} . d$ is buried depth of shield tunnel, m. $q_{0}$ is ground surface loading, $\mathrm{kPa}$.

But, when consider soil arch effect in the roof of tunnel, the calculation formula of shield tunnel vertical rock pressure as follows.

$q=\gamma_{m} h_{p}$

Where, $h_{p}$ is high pressure arch of tunnel roof, m, and other symbols with the former.

Next, the analysis of horizontal surrounding rock pressure in shield tunnel. As shown in Fig. 3, the horizontal surrounding rock pressure is consists of two parts trectangular distribution load $p_{1}$ and triangular distributed load $p_{2}$. Obviously, $p_{1}$ is caused by vertical rock pressure in the roof of shield tunnel, and $p_{2}$ is horizontal surrounding rock pressure on the side of the tunnel that caused by soil dead weight and start from tunnel roof. Usually, for the built shield tunnl, the horizontal coefficient of lateral earth pressure should according to static earth pressure coefficient.

$\left\{\begin{array}{l}p_{1}=q K_{0} \\ p_{2}=\gamma(z-d) K_{0}\end{array}\right.$

Where, $K_{0}$ is static earth pressure coefficient, other symbols with the former.

\subsection{The calculation of rock pressure after excavation.}

After the tunnel affected by excavation, shield tunnel original stress state of equilibrium was broken, especially this deep buried shield tunnel need to consider the soil arch effect. The soil arch effect will be destroyed due to the disturbance, which will lead to large changes in shield tunnel surrounding rock pressure. Firstly, with the disappearance of soil arch effect, the height of the soil column will be changed from $h_{p}$ to $d$, so the vertical rock pressure in the roof of shield tunnel will increase, also the horizontal surrounding rock pressure will be also changed.

According to the previous analysis, the calculation method of vertical surrounding rock pressure affected by excavation is farely clear, and can calculate it according to formula (8). But, the mechanism of the horizontal surrounding rock pressure is more complicated. Therefore, based on the above analysis, the horizontal surrounding rock pressure of existing shield tunnel that located in excavation disturbed zone to was researched, considered mainly influence factors during the research is horizontal distance between tunnel and foundation pit, and deformation of supporting structure.

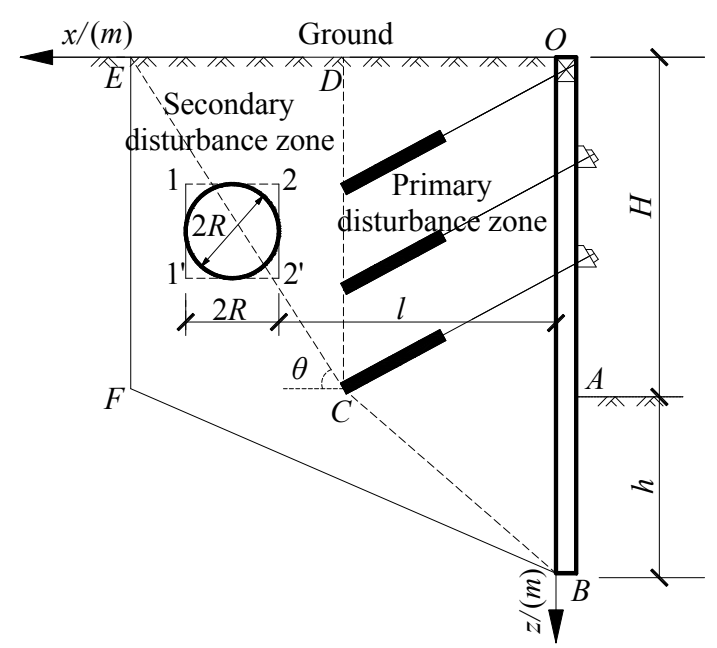

Fig. 4 The diagram of existing tunnel and pit

As shown in Fig. 4, the side wall of shield tunnel with circular cross section is equivalent to 1-1'and 2$2^{\prime}$. Based on formula (6), the formula is derivated that horizontal earth pressure calculation of shield tunnel impressed by excavation, as follow.

$p=p_{a 0}-k_{a k} \cdot \delta_{h} \cdot v(z)$

Where, $p$ is horizontal earth pressure of 1-1'and 22 ' in both sides of shield tunnel, $\mathrm{kPa}$. $p_{a o}$ is the static earth pressure at the calculation point of surface 11 'and 2-2', $\mathrm{kPa}$. Then, other symbols in formula (6) are similar with the former.

\section{ANALYSIS OF ENGINEERING EXAMPLE}

\subsection{The engineering situation.}

A excavation near existing shield tunnel in Kunming, the supporting perimeter is $639 \mathrm{~m}$, the side of near shield tunnel excavation is $160 \mathrm{~m}$, excavation depth is $18.9 \mathrm{~m}$, and the excavation use pile anchor supporting structure. Supporting pile top have a slope, the slope ratio is $1: 0.3$, platform width is $1.0 \mathrm{~m}$, dameter $1.2 \mathrm{~m}$ and horizontal spacing is $2 \mathrm{~m}$. Besides, a total of five rows of anchor, the angle of first row is $20^{\circ}$ and remaining four rows is $25^{\circ}$, the horizontal 
projected length of the bottom row is $21.6 \mathrm{~m}$, the distance of the anchor end to ground is $26.6 \mathrm{~m}$. Subway tunnel near the foundation pit is a double line circular section shield tunnel, and when foundation pit excavation the tunnel has been opened to traffic conditions. The tunnel depth is $12.5 \mathrm{~m}$, the external diameter is $6.2 \mathrm{~m}$, and the lining ring width is $1.2 \mathrm{~m}$. Above the tunnel is an inter city trunk road, the west is a wasteland and the east is foundation pit. The clear distance between excavation line and subway tunnel of the left line is $35 \mathrm{~m}$, and the clear distancbetween excavation line and subway tunnel of the right line is $41.2 \mathrm{~m}$, specifically situation as shown in Fig. 5.

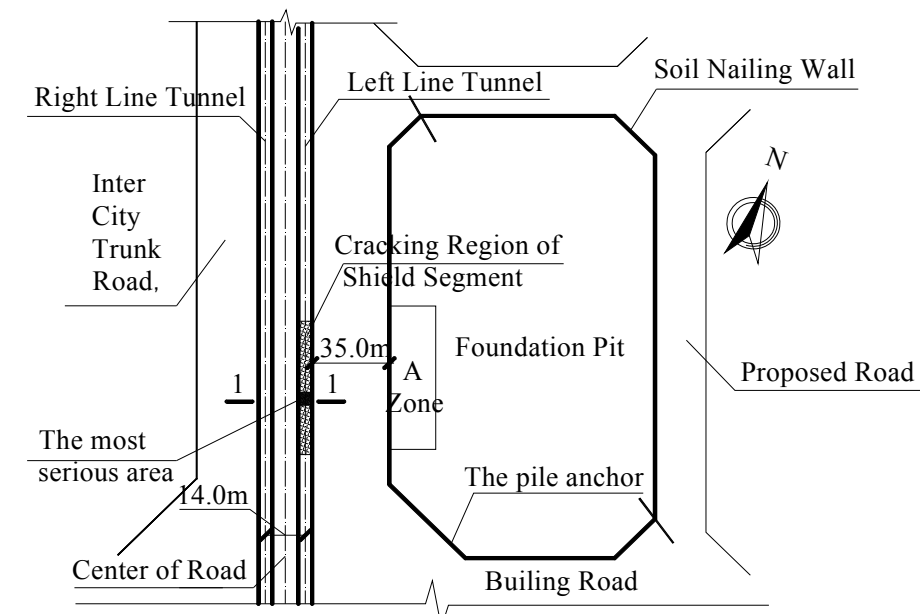

(a) The foundation pit and tunneling plane diagram

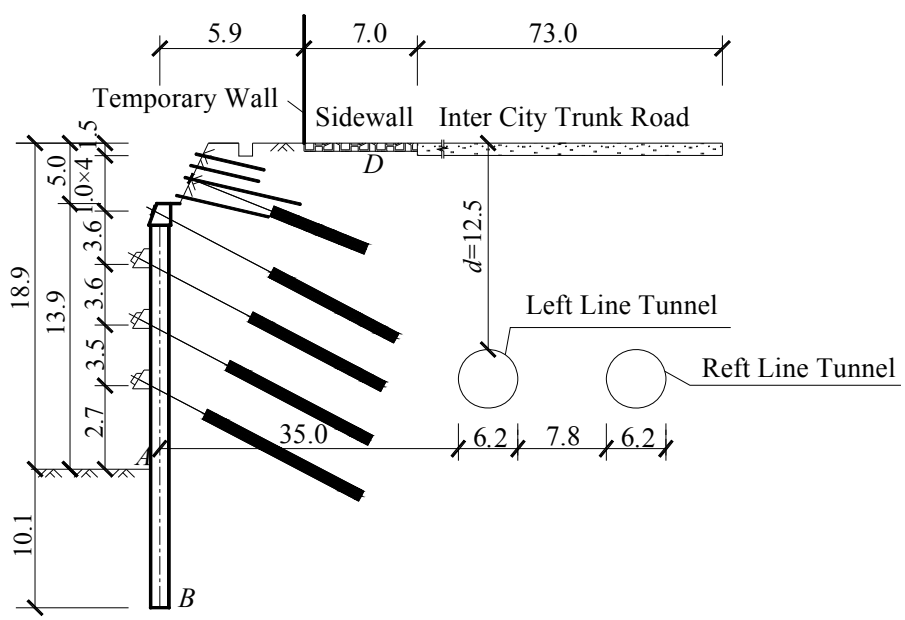

(b) The sectional 1-1 diagram

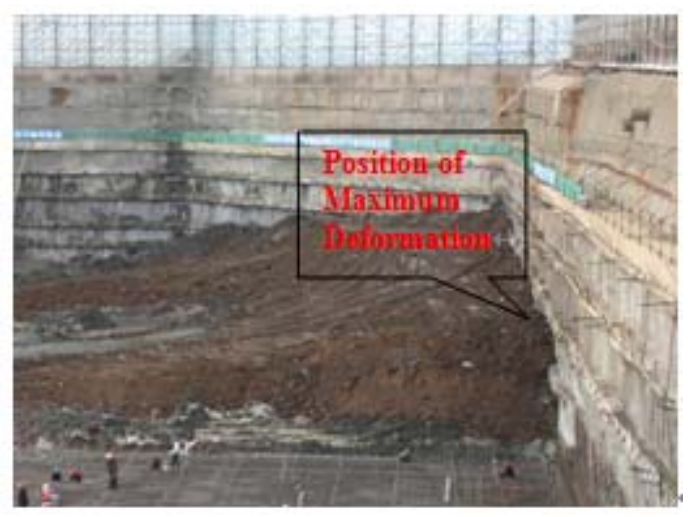

(c) The scene photos of foundation pit

Fig. 5 The diagram of foundation pit and tunnel space locations

According to the field data, When the foundation pit is completed and basement floor under construction, monitoring found the foundation pit appear larger deformation in Fig. 5(a) in the A zone. The largest deformation position appears in the fourth and fifth rows of anchor cable as show in Fig. 5(c), and the maximum deformation of the field monitoring is $103 \mathrm{~mm}$. After the dangerous situation, the construction company take timely actions to deal with it.

Next, construction company and other relevant units work together to do a survey that foundation pit surrounding roads and existing shield tunnel damage. The survey results are as follows, firstly, some cracks appear on the main road near west side of foundation pit, and rack along the north-south direction, but the road haven't found obvious subsidence. When check subway tunnel in the larger of foundation pit deformation, left line tunnel in the region found some segments (NO. 222, 234, 235, 236,238 ) has been appear obvious horizontally cracks on the tunnel left side, also found some circumferential cracks and the most width is $20 \mathrm{~mm}$, but on the other side damage to the lighter, the scene photograph shown in Fig. 6. Besides, the right line tunnel don't affected by excavation, haven't found cracks on the segment.

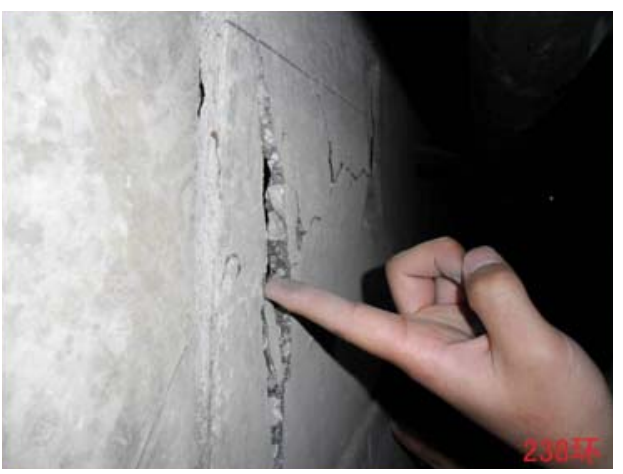

Fig. 6 The scene photos of segment broken 
For the above accident scene investigation, and select the most serious damage profile 1-1 analysis. The basic situation is shown in Fig. 5(b), and the soil parameters are shown in Table 1

Table 1 The sectional 1-1 soil information

\begin{tabular}{lllll}
\hline Name & $\begin{array}{l}\text { Thickness } \\
(\mathrm{m})\end{array}$ & $\begin{array}{l}\text { Natural } \\
\text { gravity } \\
\left(\mathrm{kN} / \mathrm{m}^{3}\right)\end{array}$ & $\begin{array}{l}\text { Cohesion } \\
(\mathrm{kPa})\end{array}$ & $\begin{array}{l}\text { Internal } \\
\text { friction } \\
\text { angle }\left({ }^{\circ}\right)\end{array}$ \\
\hline (1) silt clay & 2.0 & 18.6 & 64.4 & 6.9 \\
(2) gravel & 10.1 & 18.2 & 47.1 & 13.6 \\
clay & 5.0 & 18.2 & 54.6 & 8.5 \\
(3) clay & & 20.1 & 62.4 & 25.2 \\
(3)-2 gravel & 0.8 & 18.2 & 54.6 & 8.5 \\
sand & 1.2 & 20.2 & 40.9 & 13.8 \\
(3) clay & 1.4 & 18.2 & 54.6 & 8.5 \\
(3)-1 silt & 3.3 & 20.2 & 40.9 & 13.8 \\
(3) clay & 3.1 & 18.2 & 54.6 & 8.5 \\
(3)-1 silt & 5.3 & & & \\
(3) clay & & & & \\
\hline
\end{tabular}

The influence range of foundation pit excavation. Frist, the average of internal friction angle by soil layer thickness is $\varphi_{m}=11.2^{\circ}$, so can get the profile 1-1 is $\theta=45^{\circ}+\varphi_{m} / 2=50.6^{\circ}$. Therefore, according to the active disturbance area boundary assumption, the excavation disturbed zone boundary can be determined to BFE as shown in Fig. 7. And the distance between $E F$ face and foundation pit is $L=21.6+26.6 \times \tan 39.4^{\circ}=43.4 \mathrm{~m}$.

According to the criteria in the paper, the distance between distal left line tunnel and the foundation pit is $35+6.2=41.2 \mathrm{~m}<\mathrm{L}=43.4 \mathrm{~m}$, thus the left line tunnel will be affected by excavation. But, the distance between proximal the right line tunnel and the foundation pit is $41.2+7.8=49.0 \mathrm{~m}>\mathrm{L}=43.4 \mathrm{~m}$, so the left line tunnel will be not affected by excavation. Obviously, this paper puts forward the excavation disturbance zone assumptions and criterions are consistent with the field investigation.

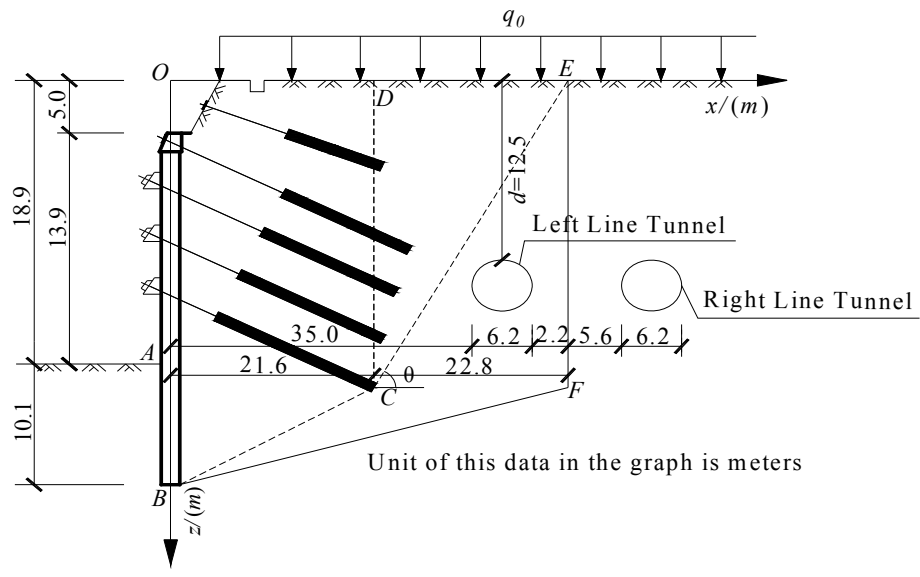

Fig. 7 The calculation diagram of shield tunnel

\subsection{Calculation of left line tunnel surrounding rock pressure before excavation.}

The left line shield tunnel buried depth is $12.5 \mathrm{~m}$, more than the tunnel outer diameter $6.2 \mathrm{~m}$, and the overlaying soil has a large shear strength, so need to consider the soil arch effect. Due to this overlaying soils are cohesive soil, need to be equivalent according to the method of literature (Cao Jing et al, 2015), the weighted average of the equivalent internal friction angle to overlaying soils is $\varphi_{D m}=30.2^{\circ}$, the weighted average value of gravity to this overlaying soils is $18.3 \mathrm{kN} / \mathrm{m}^{3}$. Thus, according to the geometric relationship as shown in Fig. 4, derived half of the width to down-sliding mass is $a_{1}$.

$$
a_{1}=R \cdot \cot \left(\left(45^{\circ}+\frac{\varphi_{D m}}{2}\right) / 2\right)=5.36 m
$$

The $a_{1}$ substituted into the formula (7), can get $h_{p}=5.36 / \tan 30.2^{\circ}=9.2 \mathrm{~m}$. Therefore, according to the formula (9), it can be obtained the vertical rock pressure before excavation $\mathrm{q}=168.4 \mathrm{kPa}$. Besides, the horizontal rock pressure can be calculated according to the formula (10), the calculation results are shown in Fig. 8.

\subsection{Calculation of left line tunnel surrounding rock pressure after excavation.}

According to the empirical formula in literature (Zhu Hehua, 2010), the deformation of supporting structure when active limit equilibrium state is reached in Rankine active destroying $v_{a}$ is $150 \mathrm{~mm}$, and the deformation of supporting structure in the corresponding range of the tunnel is $103 \mathrm{~mm}$. From Table 1, The tunnel passes through 3 layers soil, and it weighted average indexs are $\gamma_{m 1}=18.4 \mathrm{kN} / \mathrm{m}^{3}$, $c_{m 1}=55.6 \mathrm{kPa}, \varphi_{m 1}=10.70^{\circ}$.

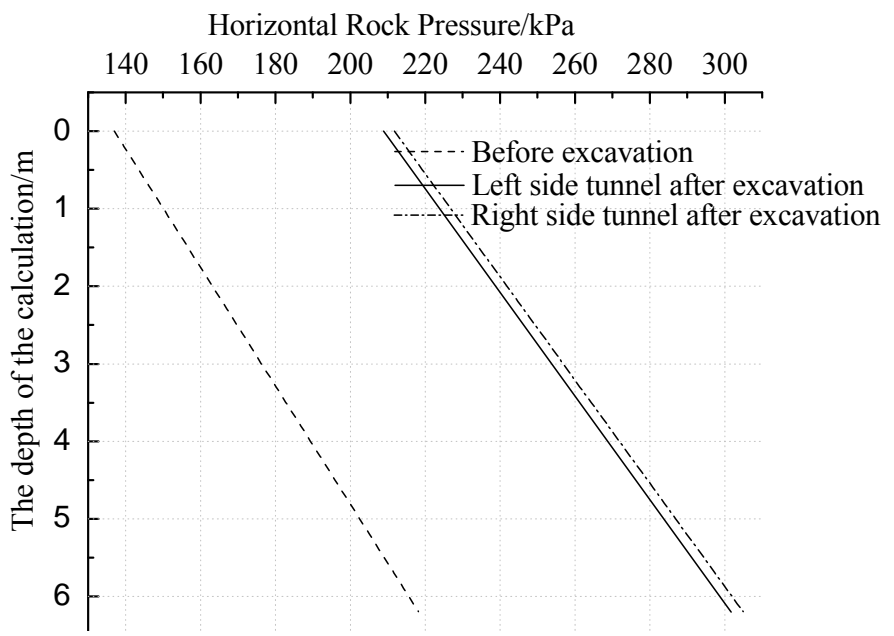

Fig. 8 The horizontal tunnel surrounding rock pressure before and after excavation 
Therefore, based on the theory proposed in this paper, firstly, the vertical rock pressure after excavation is analyzed. From the above analysis, it can be known that the vertical rock pressure before excavation was considered to the influenced by the soil arch effect, the effect of external load is not considered in the calculation. After foundation pit excavation, as shown in Fig. 7, the left line tunnel is location in the excavation disturbance zone, it supporting structure deformation is very large after excavation, and the road surface outside the foundation pit has obvious tension cracks. Thus, it indicates that this soil around the left line tunnel has obvious displacement toward foundation pit, so the soil arch effect will be disappeared. At this time, the vertical rock pressure need to calculate the tunnel roof overlying soil and ground load. So the calculation result is $\mathrm{q}=264.1 \mathrm{kPa}$, compared to the before excavation $\mathrm{q}=168.4 \mathrm{kPa}$ was increased $95.7 \mathrm{kPa}$. In addition, both sides of the horizontal shield tunnel excavation rock pressure after excavation should according to formula (11), it distribution as shown in Fg. 8 .

As shown in Fig. 8, on both sides of the tunnel, the surrounding rock pressure at the calculation point were increased to $70 \sim 88 \mathrm{kPa}$, and the main reason is the soil arching effect disappeared lead to the vertical rock pressure increasing. Besides, the horizontal surrounding rock pressure increases amount was different on both sides of the tunnel, the surrounding rock pressure is smaller on the left side of tunnel (near the foundation pit side). Of those, left and right sides of the tunnel surrounding rock pressure difference is $2.5 \sim 3 \mathrm{kpa}$. Because the tunnel left side is near to the foundation pit, which is more influenced by the excavation.

These changes for the original foundation pit before excavation, it equivalent to a larger increase in the vertical pressure, while the horizontal pressure increased slightly, the ellipsoid deformation trend of shield tunnel horizontal stretch, vertical compression will be exacerbated, it consistent with the results of field investigation. Secondly, the tunnel left side more influenced by the excavation, and the side damage situation is more seriously, it also matches field investigation.

In a word, for the deep shield tunnel, excavation foundation pit near it, the additional deformation mainly caused by the soil arch effect was destroyed led to the vertical and horizontal surrounding rock pressure increasesing.

\section{CONCLUSION}

Based on the above analysis can be obtained this conclusions.

(1) Based Rankine earth pressure theory, The calculation method of earth pressure in disturbed area was put forward, which can consider the factors of support structure deformation, and also given the corresponding calculation expressions.

(2) Excavation the foundation pit near existing shield tunnel, when the shield tunnel is located in the excavation disturbed zone, the existing shield tunnelwill have a corresponding influence on the stress and deformation by the excavation, otherwise will have no affected by excavation. Besides, when the shield tunnel is located in the excavation disturbed zone, the change of tunnel surrounding rock stress and deformation are mainly related to the depth of tunnel, horizontal distance between tunnel and foundation pit, ground loading condition and so on.

In a word, based on the analysis method of surrounding rock pressure put forward in the paper was basically consistent with the field condition, these conclusions have practical value in the research and practical engineering of the approaching construction. But, due to the lack of field data, and the influence factors are multiple in the process of excavation, the accuracy and completeness of method are still need to be further studied and solved.

\section{ACKNOWLEDGMENTS}

Corresponding auther: Cao Jing(1966-), Male, Hmong, Doctor, Professor, mainly engaged in the research of environmental geotechnical engineering. E-mail: cjlb117@sina.com.

The authors would like to thank the Key Project of the National Natural Science Foundation(NO. 51234004) for providing the financial support to conduct this research.

\section{REFERENCES}

Cao Jing, Pu Qiongxiang, Liu Haiming, et al. Analysis of influence of asymmetric filled soil on soil arch effect for open cut tunnel[J]. Rail Way Engineering, 2015, 7: 31-34.

Ding Yongchun, Dai Bing, Wang Jianhua, et al. Monitoring and Analysis of Deep Foundation Pit nearby shield tunnel[J]. Journal of Beijing University of Technology, 2008, 34(05): 492-498.

Huang Xiang, Huang Hongwei, Zhang Dongmei. Longitudinal Deflection of Existing Shield Tunnels Due to Deep Excavation[J]. Chinese Journal Geotechnical Engineering, 2012, 34(7), 1241-1250.

Liang Fayun, Chu Feng, Song Zhu, et al. Centrifugal Model Test Research on Deformation Behaviors of Deep Foundation Pit Adjacent to Metro Stations[J]. Rock and Soil Mechanics, 2012, 33(3): 657-664.

Ling Hao, Qiu Wen-ge, Sun Bing, et al. Study of Adjacent Construction of Two Tube Shield Tunnels by Centrifugal Model Test[J]. Rock and Soil Mechanics, 2010, 31(9): 28492853. 
Ou Mingxi, Liu Xinrong, Shi Janxun. Earth Pressure Applied in Retaining Wall of Deep Foundation Pit Considering Influence of Unloading and Deformation[J]. Journal of Central South University(Science and Technology), 2012, 43(2), 0669-0675.

Shen Hui, Luo Xianqi, Li Ye, et al. Numerical Simulation and Analysis of Influence of Deep Excavation on Adjacent Subway Station[J]. Chinese Journal of Underground Space and Engineering, 2011, 7(5): 1018-1024.

Wang Ning. Kranz Method on Inner Stability of Anchorreinforced Structure[J]. Exploration Engineering(Rock and Soil Drilling and Tunneling), 2008, 3: 37-38.

Wang Yanyan, Mo Haihong. Analysis on the Displacement of Metro Tunnel and Metro Station around the Excavation[J]. Science Technology and Engineering, 2011, 11(24), 58545857.

Wei Gang, Zhao Chengli, Cai Lulu. Mechanism of Foundation Pit Excavation Impact on Existing Nearby Shield Tunnel[J]. Foundation and Structure Engineering, 2013, 31(6): 141147.

Xu Huang, Helmut F.Schweiger, Hongwei Huang. Influence of Deep Excavations on Nearby Existing Tunnels[J]. International Journal of Geomechanics, 2013, 13: 170-180.

Zai Jinmian, Mei Guoxiong. Study on Calculation Method of Earth Pressure Considering Displacement and Time Effect[J]. Journal of Nanjing Architectural and Civil Engineering Institute, 2002, 1: 1-5.

Zhang Limin, Yang Geng. Analysis on Anchor Engineering for the Foundation Pit of Chengdu People's Shopping Mall[J]. Chinese Journal Geotechnical Engineering, 1992, 14(2): 078-081.

Zhang Zhiguo, Zhang Mengxi, Wang Weidong. Two-stage Method for Analyzing Effects on Adjacent Metro Tunnels Due to Foundation Pit Excavation[J]. Rock and Soil Mechanics, 2011, 32(7): 2085-2092.

Zhou Mengjia, Song Er-xiang. Overall Stability Analysis of Plie-anchor Supporting System for Deep Excavations[J]. Northwestern Seismological Journal, 2011, 33(s): 132-137.

Zhu Hehua. Underground Construction[M]. Version 2, Beijing: China Building Industry Press, 2010. 